\title{
Dietary pattern analysis reveals key food groups contributing to the successful exchange of saturated with unsaturated fatty acids in healthy men
}

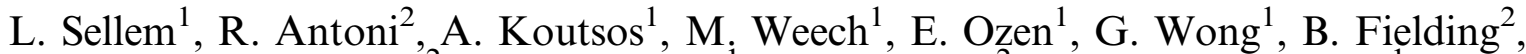 \\ M.D. Robertson ${ }^{2}$, K. G. Jackson ${ }^{1}$, B. A. Griffin ${ }^{2}$ and J. A. Lovegrove ${ }^{1}$ \\ ${ }^{1}$ Hugh Sinclair Unit of Human Nutrition, University of Reading, Whiteknights, Reading, RG6 6AP, UK and \\ ${ }^{2}$ Department of Nutritional Sciences, Faculty of Health and Medical Sciences, University of Surrey, Guildford, UK
}

Reducing dietary saturated fatty acids (SFA) to under 10\% of total energy is a key strategy for cardiovascular disease (CVD) prevention in the UK. Recent evidence suggests replacing SFA with monounsaturated (MUFA) or polyunsaturated (PUFA) fatty acids could lead to a greater CVD risk reduction compared to a replacement with carbohydrates ${ }^{(1)}$. To assess the effects of replacing dietary SFA with unsaturated fatty acids on variability in fasting serum low-density lipoprotein cholesterol, 100 healthy men $\left(30-65 \mathrm{y} ; 19-30 \mathrm{~kg} / \mathrm{m}^{2}\right)$ participated in a sequential dietary intervention study (Reading, Imperial, Surrey Saturated fat Cholesterol Intervention (RISSCI) study), following two 4-week isoenergetic diets with high ( $18 \%$ total energy) and lower ( $\leq 10 \%$ total energy) SFA, with MUFA/PUFA replacing SFA in the latter. The two diets were designed using data from the NDNS years $1-4$ rolling programme among men aged 19-64 years (2014) and a previous dietary intervention study "DIVAS" (2), identifying the following food groups as sources of exchangeable fats: butter/spreads, oils, full-fat and low-fat dairy foods, and snacks. Analysing daily nutrient intakes in a subsample from the same cohort of participants confirmed the two diets both broadly achieved their nutritional targets ${ }^{(3)}$. Thus, the current analysis aimed to investigate the impact of implementing the exchange of SFA for MUFA/PUFA to overall dietary patterns.

Dietary intake from 4-day weighed diet-diaries was categorised into 135 food groups. Differences in the overall dietary patterns of the intervention diets and intake of these specific food groups were assessed by orthogonal partial least square discriminant analysis (OPLS-DA) and Fisher tests respectively. Goodness of fit and predictability of the model were assessed by the $R^{2} Y$ and $Q^{2}$ values, respectively, and permutation tests ( $n=1,000$ permutations) were used to assess the statistical significance of the model.

The OPLS-DA revealed a clear difference in dietary patterns between the two diets $\left(\mathrm{R}^{2} \mathrm{Y}=0.899\right.$ and $\mathrm{Q}^{2}=0.743$, empirical $\mathrm{p}$-values $\mathrm{R}^{2} \mathrm{Y}: \mathrm{p}<0.001(0 / 1000)$ and $\left.\mathrm{Q}^{2}: \mathrm{p}<0.001(0 / 1000)\right)$. As indicated by the corresponding predictive loading values ( $\left.\mathrm{p}(\mathrm{corr})\right)$, this separation was driven by the foods supplied to the participants to facilitate the dietary fat exchange including solid animal fats (e.g. butter), high-fat cheese, full-fat yogurts and biscuits in the high-SFA diet ( $\mathrm{p}(\mathrm{corr})=-0.87,-0.78,-0.29$ and -0.24 , respectively), and plant-based spreads, low-fat cheese, PUFA-rich oils, nuts, low-fat yogurt and savoury snacks (e.g. crisps) in the lower-SFA diet (p (corr) $=0.78,0.72,0.67,0.62,0.33$ and 0.30 , respectively). Furthermore, there were significant differences in the level of consumption of the food groups (listed above) between the two diets $(\mathrm{p}<0.05)$.

These findings provide evidence that the foods containing the exchangeable fat were effective in achieving the dietary fat exchange. They also support compliance to the dietary advice, without significantly changing other dietary components such as meats, fish, fruits and vegetables, or carbohydrate sources.

1. Hooper L, Martin N, Abdelhamid A et al. (2015) Cochrane Database Syst Rev

2. Weech M, Vafeiadou K, Hasaj M et al. (2014) J Nutr 114, 846-855

3. Antoni R, Sellem L, Koutsos A et al. (2019) Proc Nutr Soc 78 